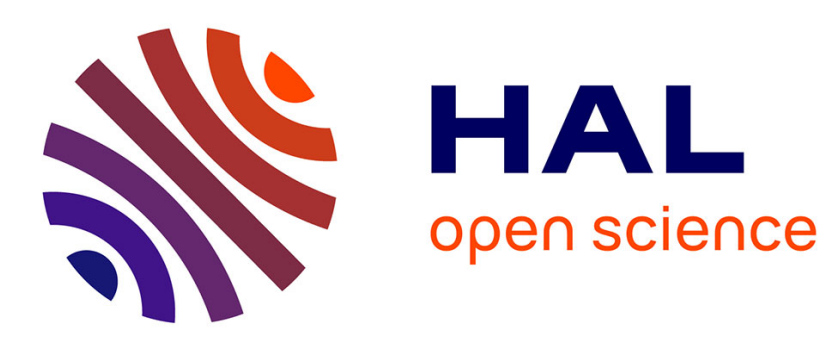

\title{
Threshold currents to move domain walls in films with perpendicular anisotropy
}

D. Ravelosona, S. Mangin, J. A. Katine, Eric E. Fullerton, B D Terris

\section{To cite this version:}

D. Ravelosona, S. Mangin, J. A. Katine, Eric E. Fullerton, B D Terris. Threshold currents to move domain walls in films with perpendicular anisotropy. Applied Physics Letters, 2007, 90, 10.1063/1.2450664 . hal-02104734

\section{HAL Id: hal-02104734 \\ https://hal.univ-lorraine.fr/hal-02104734}

Submitted on 13 May 2019

HAL is a multi-disciplinary open access archive for the deposit and dissemination of scientific research documents, whether they are published or not. The documents may come from teaching and research institutions in France or abroad, or from public or private research centers.
L'archive ouverte pluridisciplinaire HAL, est destinée au dépôt et à la diffusion de documents scientifiques de niveau recherche, publiés ou non, émanant des établissements d'enseignement et de recherche français ou étrangers, des laboratoires publics ou privés. 


\section{Threshold currents to move domain walls in films with perpendicular anisotropy}

Cite as: Appl. Phys. Lett. 90, 072508 (2007); https://doi.org/10.1063/1.2450664

Submitted: 18 October 2006 . Accepted: 08 January 2007 . Published Online: 16 February 2007

D. Ravelosona, S. Mangin, J. A. Katine, Eric E. Fullerton, and B. D. Terris

View Online

\section{ARTICLES YOU MAY BE INTERESTED IN}

Current-induced domain wall motion in a nanowire with perpendicular magnetic anisotropy Applied Physics Letters 92, 202508 (2008); https://doi.org/10.1063/1.2926664

Micromagnetic analysis of current driven domain wall motion in nanostrips with perpendicular magnetic anisotropy

Journal of Applied Physics 103, 07 E718 (2008); https://doi.org/10.1063/1.2830964

The motion of $180^{\circ}$ domain walls in uniform dc magnetic fields

Journal of Applied Physics 45, 5406 (1974); https://doi.org/10.1063/1.1663252

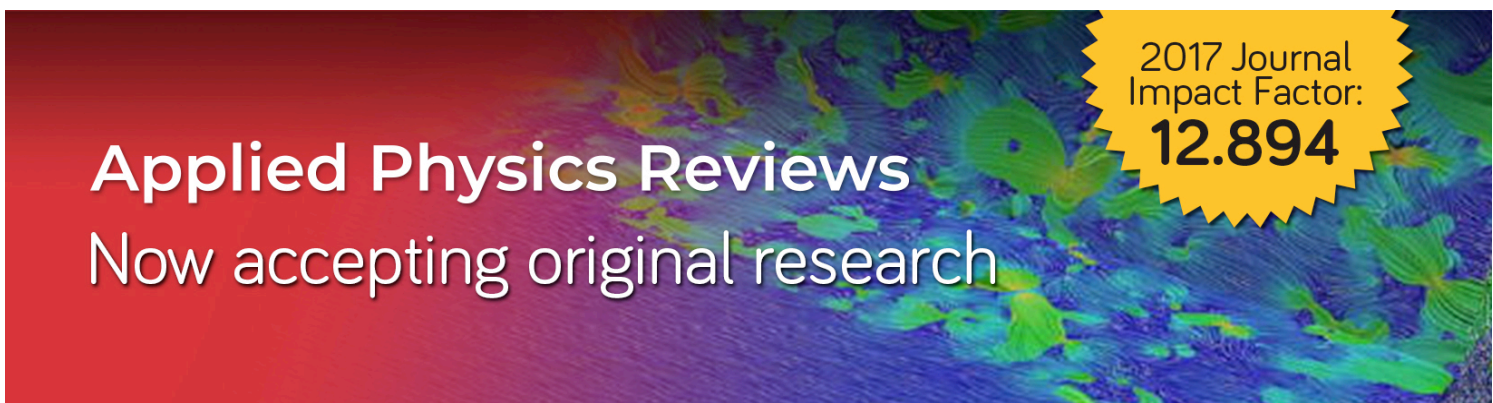

Appl. Phys. Lett. 90, 072508 (2007); https://doi.org/10.1063/1.2450664

90,072508

(c) 2007 American Institute of Physics. 


\title{
Threshold currents to move domain walls in films with perpendicular anisotropy
}

\author{
D. Ravelosona ${ }^{\text {a) }}$ \\ San Jose Research Center, Hitachi Global Storage Technologies, 3403 Yerba Buena Road, San Jose, \\ California 95135 and Institut d'Electronique Fondamentale, UMR CNRS 8622, Université Paris Sud, 91405 \\ Orsay Cedex, France \\ S. Mangin \\ San Jose Research Center, Hitachi Global Storage Technologies, 3403 Yerba Buena Road, San Jose, \\ California 95135 and LPM, U.H.P-Nancy I, B.P 239 F-54506 Vandoeuvre Cedex, France \\ J. A. Katine, Eric E. Fullerton, and B. D. Terris \\ San Jose Research Center, Hitachi Global Storage Technologies, 3403 Yerba Buena Road, San Jose, \\ California 95135
}

(Received 18 October 2006; accepted 8 January 2007; published online 16 February 2007)

The authors quantify the impact of the pinning potential on the current driven domain wall depinning process in wires with spin valves having perpendicular magnetic anisotropy. By artificially controlling the strength of the pinning field $H_{p}$, the threshold current $J_{\text {th }}$ is found to decrease when reducing $H_{p}$. In addition, the efficiency of the depinning process is observed to correlate with the polarization and the damping parameter of the magnetic layer. This suggests that the engineering of pinning sites together with well suited materials provides a pathway for efficient current-induced domain wall manipulation in films with perpendicular anisotropy. (C) 2007 American Institute of Physics. [DOI: 10.1063/1.2450664]

\begin{abstract}
Although current driven domain wall (DW) motion has now been reported in many experiments ${ }^{1-13}$ and discussed in various theories, ${ }^{14-18}$ little experimental attention has been paid to the underlying origin of the threshold current $J_{\mathrm{th}}$ that is of crucial interest for low current applications to memory and sensor devices. ${ }^{19}$ As pointed out by Tatara and Kohno, ${ }^{14}$ in the limit of weak pinning and thick DWs, $J_{\text {th }}$ is expected to be proportional to the hard-axis anisotropy $K_{\perp}$. This has been demonstrated recently by Yamaguchi et al. ${ }^{12}$ for inplane magnetized $\mathrm{NiFe}$ wires where the shape anisotropy was systematically varied and by other experiments ${ }^{13}$ showing that $J_{\text {th }}$ does not depend significantly on pinning. For stronger pinning and/or narrow DWs, the threshold current is found ${ }^{14}$ to be determined by the pinning potential (e.g., intrinsic pinning sites, constrictions, and edges roughness). Recently Thiaville et al. ${ }^{15}$ demonstrated that $J_{\text {th }}$ originates from pinning only when including a nonadiabatic contribution in the Landau-Lifshitz-Gilbert equation.

To gain a better understanding of $J_{\text {th }}$ requires modifying in a controlled and systematic way not only the pinning potential but also factors such as the polarization, damping, and DW width of the magnetic layer that may influence the efficiency of the depining process. In this letter, we report experimental determination of the $J_{\text {th }}$ while artificially varying the strength of the pinning field $H_{p}$ and by using two materials with different spin transfer efficiencies. For this we use films with perpendicular magnetic anisotropy (PMA). They exhibit narrow one-dimensional (1D) DWs that strongly interact with defects, giving rise to a very rich current-induced thermally activated depinning regime, as shown recently. ${ }^{9}$

The films used in this study are spin valve structures based on either $\mathrm{Co} / \mathrm{Pt}$ (structure I) or $\mathrm{Co} / \mathrm{Ni}$ (structure II) multilayers with PMA, as shown schematically in Fig. 1(a).
\end{abstract}

${ }^{a)}$ Electronic mail: dafine.ravelosona@ief.u-psud.fr
Structure I consists of a $\mathrm{Si} / \mathrm{Si}_{3} \mathrm{~N}_{4} / \mathrm{Pt}(1 \mathrm{~nm}) / \mathrm{Au}(20 \mathrm{~nm}) /$ $\mathrm{Pt}(5 \mathrm{~nm}) /$ reference layer/Cu$(6 \mathrm{~nm}) /$ free layer $/ \mathrm{Pt}(3 \mathrm{~nm})$ multilayer film. ${ }^{9}$ The reference layer is a $[\mathrm{Co}(0.5 \mathrm{~nm}) / \mathrm{Pt}(1 \mathrm{~nm})]_{4} / \mathrm{Co}(0.5 \mathrm{~nm})$ with high anisotropy $\left(>10^{7} \mathrm{ergs} / \mathrm{cm}^{3}\right)$, while the free layer is a single $\operatorname{Co}(0.5 \mathrm{~nm})$ film exhibiting a lower PMA $\left(\sim 6 \times 10^{6} \mathrm{ergs} / \mathrm{cm}^{3}\right)$. Structure II is $\mathrm{Si} / \mathrm{Si}_{3} \mathrm{~N}_{4} / \mathrm{Pt}(1.5 \mathrm{~nm}) /$ reference layer $/ \mathrm{Cu}(4 \mathrm{~nm}) /$ free layer $/ \mathrm{Pt}(3 \mathrm{~nm}) .^{20,21}$ The reference layer is a composite of $\mathrm{Co} / \mathrm{Pt}$ and $\mathrm{Co} / \mathrm{Ni}$ multilayers $[\mathrm{Co}(0.25 \mathrm{~nm}) / \mathrm{Pt}(0.5 \mathrm{~nm})]_{5} /$ $\left.\mathrm{Co}(0.2 \mathrm{~nm})[/ \mathrm{Co}(0.1 \mathrm{~nm}) / \mathrm{Ni}(0.6 \mathrm{~nm})]_{2} / \mathrm{Co}(0.1 \mathrm{~nm})\right]$ to enhance the perpendicular anisotropy, and the free layer is a $[\mathrm{Co}(0.1 \mathrm{~nm}) / \mathrm{Ni}(0.6 \mathrm{~nm})]_{4} / \mathrm{Co}(0.2 \mathrm{~nm})$ multilayer exhibiting PMA of the same order than the free layer of structure I. We find that use of $\mathrm{Co} / \mathrm{Ni}$ magnetic multilayers (structure II) provides perpendicular anisotropy with higher giant magnetoresistance (GMR) ratios and spin-torque efficiencies when compared to $\mathrm{Co} / \mathrm{Pt}$ multilayers (structure I) ${ }^{20}$ In both cases the reference layers have a much higher anisotropy and coercive field than the free layers. All experiments were performed in field ranges where only the free layer reverses. As illustrated in Fig. 1(b), high quality of $100 \mu \mathrm{m}$ long, $0.2-1 \mu \mathrm{m}$ wide wires were fabricated by means of electron beam lithography and ion milling. Along the wire there is a series of Hall crosses. A large reservoir at the end of the magnetic wire is used to inject a single DW within the free layer into the wire. DW motion has been detected via both the GMR of the wire and extraordinary Hall effect (EHE) [see Ref. 9 for details] at the crosses. The GMR and EHE have been measured using a high sensitivity ac $(\sim 10 \mu \mathrm{A})$ Wheatstone bridge with the magnetic field $H_{a}$ applied perpendicular to the film. The dc current has been injected by using a bias $T$. As suggested by measurements on individual films, the current density has been calculated assuming independent conduction between the $\mathrm{Au}$ and the spin valve stack 
(a) Structure I

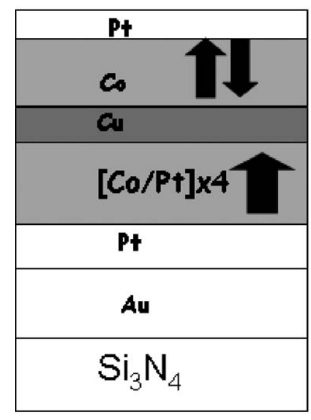

(b)

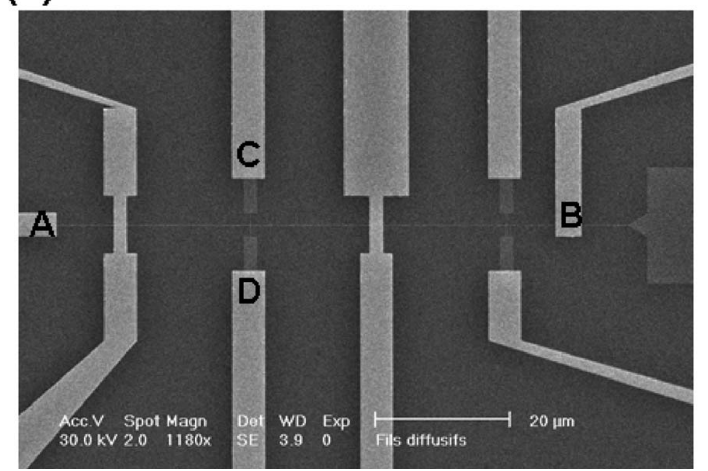

FIG. 1. (a) Schematic of the two different multilayer structures used in this experiment. (b) Scanning electron microscopy image of the magnetotransport device used in the experiments. The current is injected between electrodes $A$ and $B$ and the DW motion is detected by means of EHE in the two magnetic crosses and GMR of the wire.

in structure I (Ref. 9) and by considering a homogeneous flow in structure II. The possible shunting by the $\mathrm{Cu}$ in both structures has been neglected.

DWs in the free layer of structures I and II can be treated as $1 \mathrm{D}$ Bloch walls with a typical width $\Delta \sim 10 \mathrm{~nm}$. We previously demonstrated ${ }^{9}$ that DWs can be topologically pinned when crossing a Hall cross due to a bubblelike bending. With a 1D Bloch DW, this behavior leads to a topologically induced pinning field $H_{\text {top }}(w)=\left(\sigma / w M_{S}\right)$ at the center of the Hall cross, $w$ being the wire width, $M_{s}$ the saturation magnetization, and $\sigma$ the wall energy. The total pinning field in the Hall cross for the bent DW is $H_{p}(w)=H_{\mathrm{pi}}+H_{\mathrm{top}}(w)$, where $H_{\mathrm{pi}}$ is the intrinsic pinning field of the unpatterned film due to homogeneously distributed structural defects. Thus, by varying the width $w$ of a Hall cross $(0.2,0.5$, and $1 \mu \mathrm{m}$ ) in a same $\mathrm{Co} / \mathrm{Pt}$ (structure I) sample, we have artificially controlled the strength of the pinning field $H_{p}$. This is illustrated in Fig. 2(a) where time resolved EHE resistance at constant net field acting on the free layer $H_{\text {free }}$ is shown. We observe a systematic increase of the depinning time when the wire width is reduced. The value of $H_{p}$ is then determined by measuring the time of depinning as a function of the $H_{\text {free }}$ for each wire. This response is well described by the classical Arrhenius behavior $\tau\left(H_{\text {free }}\right)=\tau_{0} \exp \left[E\left(H_{\text {free }} / k_{B} T\right]\right.$ with $E\left(H_{\text {free }}\right)=2 M_{S} V\left(H_{p}-H_{\text {free }}\right)$, where $\tau_{0}$ is an intrinsic depinning time (approximately nanoseconds) and $k_{B} T$ the thermal energy. Figure 2(b) shows the expected $1 / w$ variation with the pinning field varying from 400 to 150 Oe for $w=0.2$ and $1 \mu \mathrm{m}$, respectively. The slope of the fit is found to be very close to the expected $\sigma / M_{S} \sim 5 \times 10^{3} \mathrm{Oe} / \mathrm{nm}$ value $(\sigma=5$
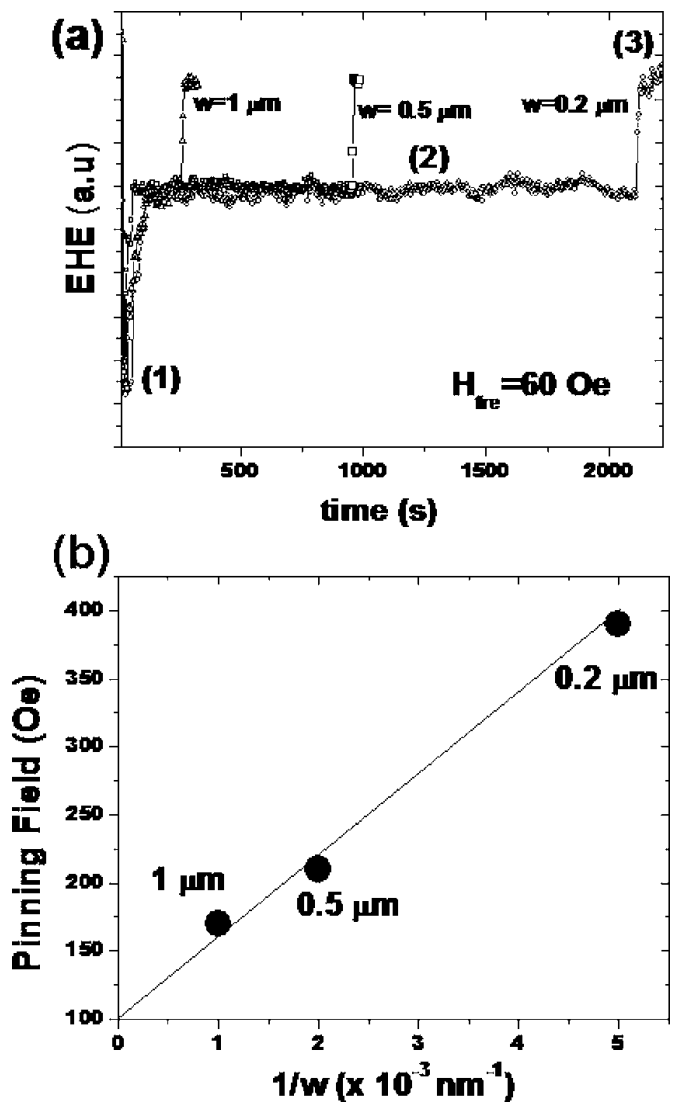

FIG. 2. (a) Typical EHE measurement as a function of time at constant field for different wire widths for structure I. Under $H_{\text {free }}=60 \mathrm{Oe}$, the DW propagates from the input of the cross (1) by discrete jumps of about $10 \mathrm{~nm}$ until it is pinned at the center of the cross (2). The DW later becomes depinned from the center to the output of the cross (3) at a later time indicated by the jump in the EHE signal. The depinning time $\tau\left(H_{\text {free }}\right)$, is given by the plateau region. (b) The pinning field $H_{p}$ as a function of the wire width deduced from the field dependence of the pinning time, as measured in (a).

$\times 10^{6} \mathrm{ergs} / \mathrm{cm}^{2}$ and $M_{S}=1500 \mathrm{emu} / \mathrm{cm}^{3}$ ), and we find $H_{p}(1 / w \rightarrow 0) \sim 106$ Oe which is consistent with the value of $H_{\mathrm{pi}}$ found in our unpatterned films.

Using the procedure described in Ref. 9, we measured the threshold current density $J_{\text {th }}$ as a function of $w$. The sample is first prepared with a DW pinned at the center of the cross CD [see Fig. 1(b)] where the pinning strength is $H_{p}(w)$, and then the current $I$ (here the electrons flow from contact A to $\mathrm{B}$ is increased in steps of $0.1 \mathrm{~mA}$ in typical time intervals of $\Delta t=1 \mathrm{~s}$ ). The motion of the DW in the Hall cross is determined from the value of the EHE resistance. We find that under the influence of the critical current density $J_{\text {th }}$ the DW is always depinned from the center to the output of the cross in the same direction as the electron flow. ${ }^{22}$ Figure 3 shows that the threshold current $J_{\text {th }}$ decreases when reducing $H_{p}$, leading to a value of $J_{\mathrm{th}} \sim 5 \times 10^{6} \mathrm{~A} / \mathrm{cm}^{2}$ for the lowest $H_{p}$. This shows that the threshold current density can be decreased by the reduction of the "extrinsic" pinning field. Interestingly, note that by extrapolating the linear behavior to $H_{p}=0$, we find $J_{\text {th }} \sim 0$. These features are consistent with the theoretical prediction for a pinning dominated mechanism ${ }^{14,18}$ due either to the strong pinning in our films or the narrow DW width. This is also consistent with a recent study, ${ }^{9}$ where nanometer scale observation of thermally as- 


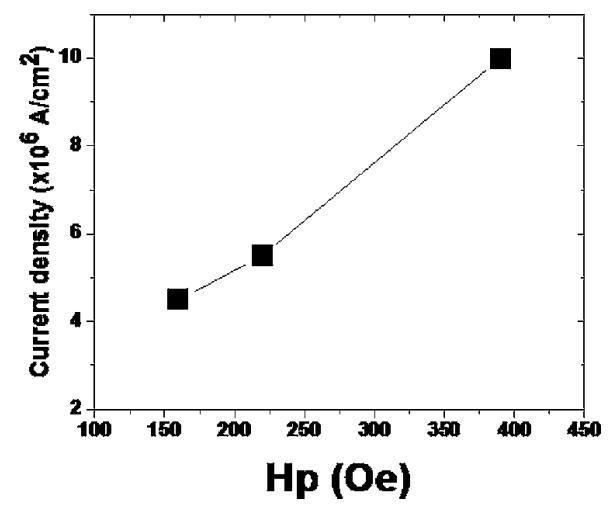

FIG. 3. Critical current density $J_{\text {th }}$ to depin the DW from the center of the Hall cross as a function of the pinning field $H_{p}$ for structure I.

sisted current driven depinning in a 200 -nm-wide wire made from the same $\mathrm{Co} / \mathrm{Pt}$ multilayer was reported. The time of depinning as a function of the net field $H_{\text {free }}$ and current density $J$ was found to follow an Arrhenius law $\tau\left(H_{\text {free }}, J\right)$ $=\tau_{0} \exp \left[E\left(H_{\text {free }}, J\right) / k_{B} T\right]$, where $E\left(H_{\text {free }}, J\right)$ is the energy barrier given by

$$
E\left(H_{\text {free }}, J\right)=2 M_{S} V\left[H_{P}-\left(A / 2 M_{S} V\right) J-H_{\text {free }}\right]
$$

and $A$ is a parameter related to the spin transfer mechanism and $V$ is the activation volume. This expression simply shows that in this thermally activated regime, the depinning threshold is governed by $H_{p}-H_{J}$, where $H_{J}=\left(A / 2 M_{S} V\right) J$ is an effective perpendicular field opposing $H_{p}$.

As demonstrated in our previous studies in current perpendicular to plane (CPP) nanopillars, ${ }^{20} \mathrm{Co} / \mathrm{Ni}$ multilayers exhibit higher GMR ratios and spin-torque efficiencies when compared to $\mathrm{Co} / \mathrm{Pt}$ multilayers. We then may expect currentinduced DW motion mechanisms to be affected when using $\mathrm{Co} / \mathrm{Ni}$ multilayers. Figure 4 shows $\ln \tau(J) / \tau(J=0)$ versus the current density $J$ for a wire width $w=200 \mathrm{~nm}$ fabricated in structures I and II. The measurements have been realized at different magnetic fields for both structures. However, since we have found that the parameters $A$ and $V$ do not depend on either $J$ or $H$, the linear behavior indicated in Fig. 4 allows determining directly the parameter $A$ for the two structures. The strongest influence of the current is observed for the $\mathrm{Co} / \mathrm{Ni}$ sample. From the fit to the experimental curves using

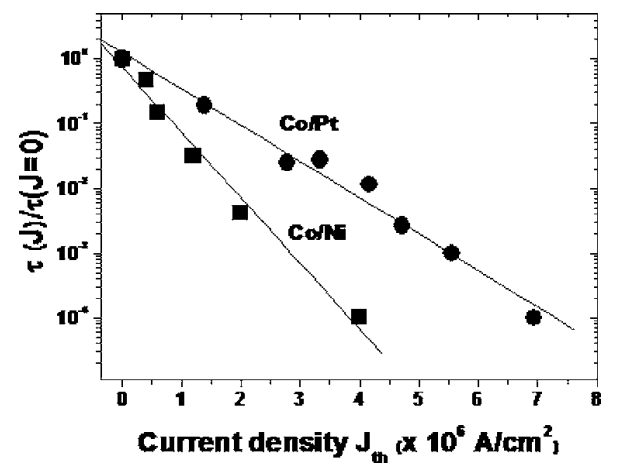

FIG. 4. Normalized time of depinning as a function of the current density for structures I (circles) and II (squares). The DW is initially pinned at the center of the Hall cross. Then a dc current $I$ is applied and the depinning time $\tau(I)$ is measured.
Eq. (1), we deduced $A(\mathrm{CoNi})=3 A(\mathrm{Co} / \mathrm{Pt})$ which shows a higher spin transfer efficiency in CoNi structures for currentinduced DW depinning process. From Eq. (1), the critical current at $T=0 \mathrm{~K}$ is given by $J_{\text {th }}=H_{p}\left(2 M_{S} V / A\right)$. It is then anticipated from recent theories ${ }^{14,18}$ that $A$ should depend on the DW width $\lambda$, the damping constant $\alpha$, and the current polarization $p$. We expect $J_{\text {th }}$ to decrease when decreasing $\lambda$ or $\alpha$ or by increasing $p$. Here, the $\mathrm{Co} / \mathrm{Pt}$ and $\mathrm{Co} / \mathrm{Ni}$ free layers exhibit similar anisotropy fields, $M_{S}$ values, and DW widths. The higher efficiency measured here is then consistent with our previous studies on CPP nanopillars ${ }^{20}$ that suggested for $\mathrm{Co} / \mathrm{Pt}$ a lower $p / \alpha$ ratio as compared to the $\mathrm{Co} / \mathrm{Ni}$ samples.

In conclusion, controlled manipulation of the pinning potential in wires with perpendicular anisotropy indicates that the critical current density for DW depinning process under spin transfer strongly depends on the strength of the pinning and the polarization and damping of the material. This suggests that a precise engineering of the pinning barrier together with more efficient materials can further decrease the critical current densities for domain wall manipulation in nanostructures. However, care will be required to design sufficient pinning potential to avoid thermal stability issues in very small elements.

${ }^{1}$ J. Grollier, P. Boulenc, V. Cros, A. Hamzic, A. Vaurs, A. Fert, and G. Faini, Appl. Phys. Lett. 83, 509 (2003).

${ }^{2}$ M. Klaui, C. A. F. Vaz, J. A. C. Bland, G. Faini, E. Cambril, and L. J. Heyderman, Appl. Phys. Lett. 83, 105 (2003).

${ }^{3}$ M. Tsoi, R. E. Fontana, and S. S. Parkin, Appl. Phys. Lett. 83, 2617 (2003).

${ }^{4}$ N. Vernier, D. A. Allwood, D. Atkinson, M. D. Cooke, and R. P. Cowburn, Europhys. Lett. 65, 526 (2004).

${ }^{5}$ A. Yamaguchi, T. Ono, S. Nasu, K. Miyake, K. Mibu, and T. Shinjo, Phys. Rev. Lett. 92, 077205 (2004).

${ }^{6}$ E. Saitoh, H. Miyajima, T. Yamaoka, and G. Tatara, Nature (London) 432, 203 (2004).

${ }^{7}$ M. Yamanouchi, D. Chiba, F. Matsukura, and H. Ohno, Nature (London) 428, 539 (2004)

${ }^{8}$ A. Yamaguchi, S. Nasu, H. Tanigawa, T. Ono, K. Miyake, K. Mibu, and T. Shinjo, Appl. Phys. Lett. 86, 012511 (2005).

${ }^{9}$ D. Ravelosona, D. Lacour, J. A. Katine, B. D. Terris, and C. Chappert, Phys. Rev. Lett. 95, 117203 (2005).

${ }^{10}$ M. Klaui, M. Laufenberg, L. Heyne, D. Backes, U. Rudiger, C. A. F. Vaz, J. A. C. Bland, L. J. Heyderman, S. Cherifi, A. Locatelli, T. O. Mentes, and L. Aballe, Appl. Phys. Lett. 88, 232507 (2006).

${ }^{11}$ M. Hayashi, L. Thomas, Ya. B. Bazaliy, C. Rettner, R. Moriya, X. Jiang, and S. S. P. Parkin, Phys. Rev. Lett. 96, 197207 (2006).

${ }^{12}$ A. Yamaguchi, K. Yano, H. Tanigawa, S. Kasai, and T. Ono, Jpn. J. Appl. Phys., Part 1 45, 3850 (2006).

${ }^{13}$ L. Thomas, M. Hayashi, X. Jiang, R. Moriya, C. Rettner, and S. S. P. Parkin, Nature (London) 443, 197 (2006).

${ }^{14}$ G. Tatara and H. Kohno, Phys. Rev. Lett. 92, 086601 (2004).

${ }^{15}$ A. Thiaville, Y. Nakatani, J. Miltat, and N. Vernier, J. Appl. Phys. 95, 7049 (2004).

${ }^{16}$ Z. Li and S. Zhang, Phys. Rev. Lett. 92, 207203 (2004).

${ }^{17}$ S. Shang and Z. Li, Phys. Rev. Lett. 93, 127204 (2004).

${ }^{18}$ A. Thiaville, Y. Nakatani, J. Miltat, and Y. Susuki, Europhys. Lett. 69, 990 (2005).

${ }^{19}$ S. S. P. Parkin, U.S. Patent No. 6834005 (21 December 2004).

${ }^{20}$ S. Mangin, D. Ravelosona, J. Katine, B. Terris, and E. E. Fullerton, Nat. Mater. 5, 210 (2006)

${ }^{21}$ D. Ravelosona, S. Mangin, Y. Lemaho, J. A. Katine, B. D. Terris, and E. E. Fullerton, Phys. Rev. Lett. 96, 186604 (2006).

${ }^{22}$ Note that as measured by using the GMR effect in the wire, we have not detected any DW injection for $J<5 \times 10^{7} \mathrm{~A} / \mathrm{cm}^{2}$ into the wire where probably edge roughness prevents DW propagation, whereas in the hall cross once depinned the DW is free. 